\title{
Предельная температура генерации микродисковых лазеров
}

\author{
(C) А.Е. Жуков ${ }^{1}$, Н.В. Крыжановская ${ }^{1}$, Э.И. Моисеев ${ }^{2,}$, М.М. Кулагина ${ }^{3}$, С.А. Минтаиров ${ }^{3}$, \\ Н.A. Калюжный ${ }^{3}$, А.М. Надточий ${ }^{2}$, М.В. Максимов ${ }^{2}$ \\ ${ }^{1}$ Национальный исследовательский университет „Высшая школа экономики“, \\ 190008 Санкт-Петербург, Россия \\ ${ }^{2}$ Санкт-Петербургский национальный исследовательский Академический университет им. Ж.И. Алфёрова \\ Российской академии наук, \\ 194021 Санкт-Петербург, Россия \\ ${ }^{3}$ Физико-технический институт им. А.Ф. Иоффе Российской академии наук, \\ 194021 Санкт-Петербург, Россия \\ ฯ E-mail: moiseev@spbau.com, krij@mail.ioffe.ru
}

Поступила в Редакцию 23 января 2020 г.

В окончательной редакции 31 января 2020 г.

Принята к публикации 31 января 2020 г.

Развита модель, которая позволяет в аналитическом виде определить пороговый ток микродискового лазера с учетом саморазогрева в зависимости от температуры окружающей среды и диаметра микролазера. Показано, что существует обусловленный саморазогревом минимальный диаметр микродиска, вплоть до которого возможно достижение лазерной генерации в непрерывном режиме при заданной температуре. Другим проявлением саморазогрева является существование предельной рабочей температуры, тем меньшей, чем меньше диаметр микролазера. Показано хорошее совпадение предсказаний модели с имеющимися экспериментальными данными.

Ключевые слова: микрорезонатор, микролазер, саморазогрев, тепловое сопротивление.

DOI: 10.21883/FTP.2020.06.49387.9354

\section{1. Введение}

Начиная с 90-х годов прошлого столетия большое внимание исследователей привлекают полупроводниковые микродисковые и микрокольцевые лазеры [1-4] приборы, в которых высокая добротность достигается за счет формирования мод шепчущей галереи, а размеры варьируются от единиц до десятков микрометров. В последние годы такие микролазеры рассматриваются в качестве одних из наиболее перспективных кандидатов для использования в системах оптической передачи данных на предельно малые расстояния (между и внутри интегральных микросхем), в том числе интегрированных с элементами транзисторной логики на кремниевых подложках [5-8].

Рабочая температура микролазера, входящего в состав оптоэлектронной интегральной схемы или размещенного в ее непосредственной близости, повышается вследствие тепловыделения элементов микросхемы. Наибольшее опубликованное значение рабочей температуры микродисковых/микрокольцевых лазеров, составляющее на сегодня $100-110^{\circ} \mathrm{C}$, было достигнуто в приборах относительно большого диаметра (30-100 мкм), тогда как с уменьшением размера снижалась и рабочая температура [9-11]. Кроме окружающей среды, микролазер подвергается воздействию тепла, выделяемого самим прибором в процессе его работы (так называемый саморазогрев). Отвод тепла от активной области микролазера может быть улучшен за счет увеличения площади прибора, что входит в очевидное противоречие с необходимостью его миниатюризации для снижения абсолютной величины рабочего тока, увеличения быстродействия, повышения плотности компоновки приборов. В связи с этим приобретает актуальность задача разработки микролазеров возможно меньшего диаметра, способных сохранять работоспособность при повышенных температурах.

Наиболее полно эффект саморазогрева к настоящему времени исследован для поверхностно-излучающих лазеров с вертикальным микрорезонатором [12-14], тогда как для микродисковых/микрокольцевых лазеров вопросы внутреннего тепловыделения, максимальной рабочей температуры и причин, ее устанавливающих, ранее не обсуждались. В настоящей работе мы описываем теоретическую модель, позволяющую найти предельную температуру лазерной генерации микродискового лазера и установить ее зависимость от размера прибора.

\section{2. Параметры модели}

Для расчета высокотемпературного поведения микролазеров в условиях саморазогрева нами были использованы параметры, ранее полученные в ходе экспериментальных исследований микродисковых лазеров $\mathrm{AlGaAs} / \mathrm{GaAs}$ с активной областью на основе плотных массивов квантовых точек (In,Ga)As (так называемых квантовых ям-точек (КЯТ) [15]), синтезируемых методом газофазной эпитаксии из металлорганических соединений. Активная область, содержащая несколько слоев КЯТ, помещается в нелегированный волноводный 


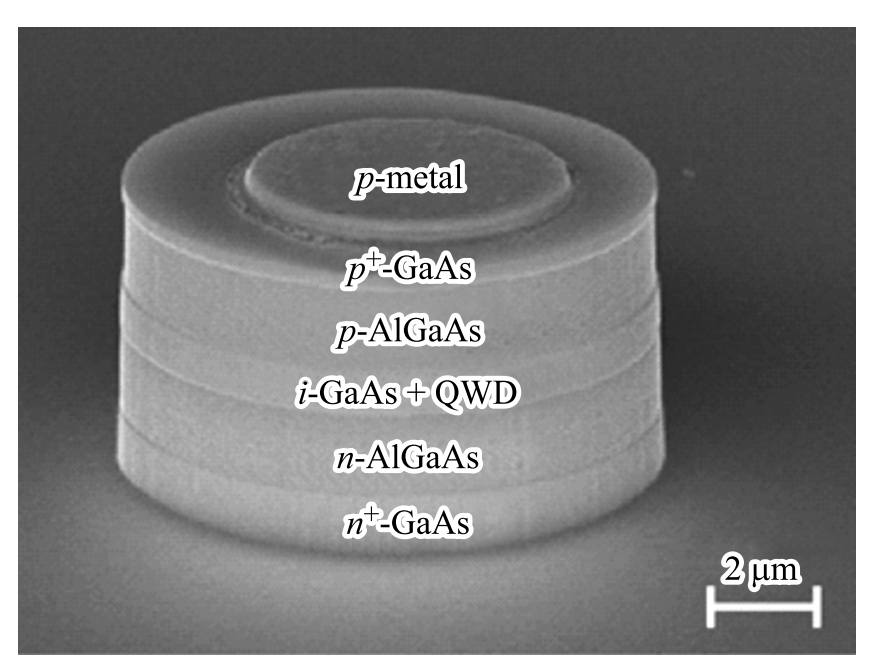

Рис. 1. Микрофотография микродискового лазера диаметром 10 мкм.

слой GaAs толщиной около 0.6-0.8 мкм, ограниченный легированными эмиттерными слоями $\mathrm{Al}_{x} \mathrm{Ga}_{1-x} \mathrm{As}$ $(x \sim 0.3-0.4)$ толщиной более 1 мкм. Микродисковые резонаторы диаметром 10-30 мкм и высотой $\sim 5$ мкм формируются с помощью глубокого (сквозь активную область) травления лазерной гетероструктуры (рис. 1). Такие микролазеры продемонстрировали наивысшую на сегодня температуру лазерной генерации в непрерывном режиме $\left(110^{\circ} \mathrm{C}[10]\right)$, частоту прямой модуляции $>6$ ГГц и безошибочную оптическую передачу данных при $30^{\circ} \mathrm{C}$ без внешней стабилизации температуры со скоростью 10 Гб/с [16].

Исследования микродисковых лазеров различного диаметра показали, что тепловое сопротивление микродискового лазера $R_{T}=\rho_{T} / S$ изменяется обратно пропорционально площади микродиска $S=\pi D^{2} / 4$, где $\rho_{T}=2.8 \cdot 10^{-3} \mathrm{~cm}^{2} \cdot \mathrm{K} / \mathrm{BT}[10]$ - удельное тепловое сопротивление. Вольт-амперная характеристика $U(I)$ приближенно описывается выражением $U=U_{0}+I R_{S}$, где $U_{0}=1.446$ B [17] - напряжение открывания диода, $R_{S}=\rho_{S} / S_{\text {eff }}$ - последовательное электрическое сопротивление, $\rho_{S}=10^{-4} \mathrm{~cm}^{2} \cdot$ Ом [17] - удельное электрическое сопротивление, $S_{\text {eff }}=\pi(D-\delta)^{2} / 4-$ эффективная площадь протекания тока, $\delta / 2=1.5$ мкм [18] толщина непроводящего поверхностного слоя вблизи боковых стенок. Пороговый ток микродискового лазера при комнатной температуре без учета саморазогрева

$$
I_{0}=\frac{\pi D^{2}}{4} J_{2}+\pi D j_{1},
$$

где первое слагаемое описывает вклад рекомбинации в объеме активной области, а второе - вклад поверхностной безызлучательной рекомбинации на боковых стенках микрорезонатора, $J_{2}=630 \mathrm{~A} / \mathrm{cm}^{2}$ и $j_{1}=0.75 \mathrm{~A} / \mathrm{cm}[17]$ - удельные значения соответствующих компонент порогового тока, нормированные на площадь и периметр микродиска. Для простоты будем полагать, что характеристическая температура порогового тока $T_{0}=90 \mathrm{~K}$, определенная для микродиска диаметром 30 мкм [10], остается неизменной при изменении $D$. Такое допущение представляется обоснованным, учитывая, что оптические потери в микродисковых лазерах малы и не обнаруживают регулярной зависимости от $D[16]$.

\section{3. Модель}

Пороговый ток микролазера $I_{\text {th }}$ увеличивается при возрастании температуры его активной области $T_{\text {act. }}$ Эта зависимость может быть описана с помощью эмпирического выражения

$$
I_{\mathrm{th}}=I_{0} \exp \left(\frac{T_{\mathrm{act}}-20^{\circ} \mathrm{C}}{T_{0}}\right),
$$

где $I_{0}$ - пороговый ток в отсутствие саморазогрева при $20^{\circ} \mathrm{C}$ - температуре, принятой за начало отсчета. При этом не имеет значения, вызван ли рост $T_{\text {act }}$ изменением температуры $(T)$ окружающей среды/теплоотвода, либо дополнительным увеличением температуры $(\Delta T)$ прибора вследствие его саморазогрева. Последний обусловлен выделением джоулева тепла $Q=U I$ и конечным тепловым сопротивлением $R_{T}$ микролазера:

$$
\Delta T=R_{T} Q
$$

При накачке постоянным током I выделяемое микролазером тепло

$$
Q \approx I U_{0}+I^{2} R_{S}
$$

Мы учли, что излучаемая микролазером оптическая мощность типично мала по сравнению с подводимой электрической мощностью.

Лазерная генерация возникает, когда ток инжекции сравнивается с пороговым током $I_{\text {th }}$. Так как величина саморазогрева сама зависит от протекающего через микролазер тока, пороговый ток с учетом (2) и (3а,б), может быть найден из условия:

$$
I_{\text {th }}=I_{0} \exp \left(\frac{T-20^{\circ} \mathrm{C}+R_{T}\left(I U_{0}+I^{2} R_{S}\right)}{T_{0}}\right)=I .
$$

Графическое решение этого трансцендентного уравнения проиллюстрировано на рис. 2, где сплошной линией показана правая часть уравнения (4), а серия штриховых линий изображает левую часть при различных значениях температуры окружающей среды. Увеличение $T$ приводит к подъему левой части (4) и, соответственно, сдвигает пороговый ток (точку пересечения кривых) в сторону бо́льших значений (рис. 3).

В области низких температур (для приведенного на рис. 3 примера вплоть до $T \approx 50^{\circ} \mathrm{C}$ ) саморазогрев оказывает незначительное влияние на пороговый ток, так что $I_{\text {th }} \approx I_{0} \exp \left(\left(T-20^{\circ} \mathrm{C}\right) / T_{0}\right)$. Однако при более высоких 
температурах пороговый ток увеличивается с ростом $T$ заметно быстрее. Существует предельная температура окружающей среды $\left(T_{\max }\right)$, при которой лазерная генерация все еще возможна. Для параметров микролазера, использованных для рис. 2 и $3, \mathrm{~T}_{\max } \approx 117^{\circ} \mathrm{C}$.

Если температура окружающей среды превышает $T_{\max }$, то левая и правая части (4) не имеют пересечений. Это означает, что лазерная генерация не будет достигнута ни при каком уровне инжекции. При предельной рабочей температуре кривые, соответствующие левой и правой частям уравнения (4), имеют в точке пересечения касание 1-го порядка. Условие равенства первых производных:

$$
\begin{aligned}
d I_{\mathrm{th}} / d I= & {\left[I_{0} \exp \left(\frac{T-20^{\circ} \mathrm{C}+R_{T}\left(I U_{0}+I^{2} R_{S}\right.}{T_{0}}\right)\right] } \\
& \times \frac{R_{T}}{T_{0}}\left(U_{0}+2 I R_{S}\right)=1,
\end{aligned}
$$

совместно с (4) позволяет определить ток инжекции $I_{\max }$, при котором происходит касание (при этом токе оптическая мощность лазерной генерации микролазера обращается в нуль), и соответствующую ему предельную температуру:

$$
\begin{gathered}
\frac{R_{T}}{T_{0}}\left(U_{0}+2 I_{\max } R_{S}\right) I_{\max }=1, \\
I_{0} \exp \left(\frac{T_{\max }-20^{\circ} \mathrm{C}+\Delta T_{\max }}{T_{0}}\right)=I_{\max },
\end{gathered}
$$

где через

$$
\Delta T_{\max }=R_{T}\left(I_{\max } U_{0}+I_{\max }^{2} R_{S}\right)
$$

мы обозначили величину саморазогрева при токе $I_{\max }$.

Решение квадратного уравнения (6a):

$$
I_{\max }=\frac{-U_{0}+\sqrt{U_{0}^{2}+8 \frac{T_{0} R_{S}}{R_{T}}}}{4 R_{S}} .
$$

Из (6б) следует, что

$$
T_{\max }=T_{0} \ln \left(I_{\max } / I_{0}\right)-\Delta T_{\max }+20^{\circ} \mathrm{C} .
$$

При значениях параметров, характерных для рассматриваемых микролазеров, второе слагаемое, стоящее под знаком корня в (7a), заметно превышает напряжение открывания и, таким образом,

$$
\begin{gathered}
I_{\max } \approx \sqrt{\frac{T_{0}}{2 R_{T} R_{S}}}, \\
\Delta T_{\max } \approx I_{\max }^{2} R_{S} R_{T} \approx \frac{T_{0}}{2}, \\
T_{\max } \approx \frac{T_{0}}{2}\left[\ln \left(\frac{T_{0}}{2 R_{T} R_{S} I_{0}^{2}}\right)-1\right]+20^{\circ} \mathrm{C} .
\end{gathered}
$$

Как следует из (8б), максимальное значение саморазогрева $\Delta T_{\max }$, при котором еще сохраняется лазерная генерация, приближенно равно половине характеристической температуры.

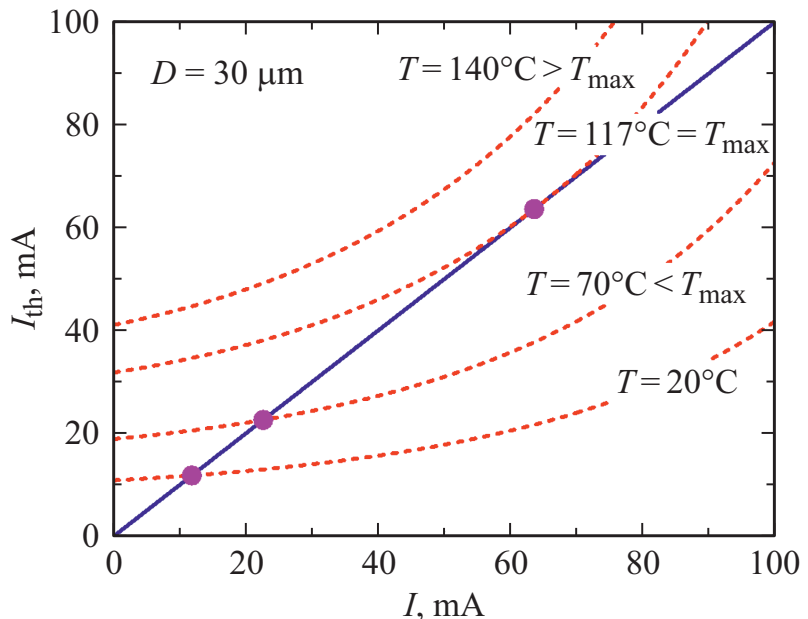

Рис. 2. Графическое решение порогового условия микролазеpa $(D=30$ мкм): сплошная линия - ток инжекции (правая часть (4)), штриховые линии - пороговый ток с учетом саморазогрева (левая часть (4)) при различной температуре окружающей среды $T$.

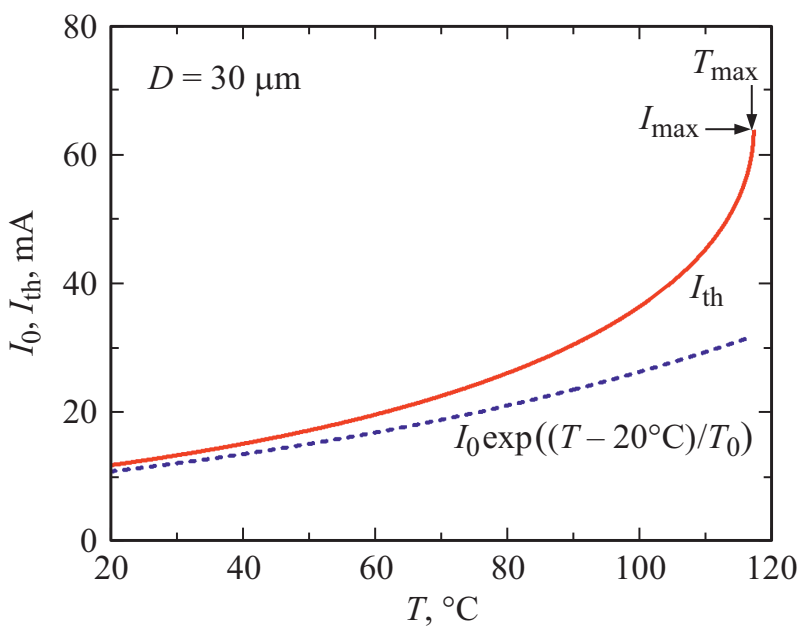

Рис. 3. Зависимость порогового тока микролазера $(D=30$ мкм $)$ от температуры окружающей среды с учетом (сплошная линия) и без учета (штриховая линия) саморазогрева.

\section{4. Зависимость пороговых характеристик от диаметра микролазера}

Обратимся теперь к обсуждению того, как размер микролазера влияет на пороговые характеристики в условиях саморазогрева. Сначала обсудим случай, когда температура окружающей среды составляет $20^{\circ} \mathrm{C}$. На рис. 4 показана зависимость от диаметра максимального тока лазерной генерации $\left(I_{\max }\right)$, порогового тока при $20^{\circ} \mathrm{C}$ в случае, когда саморазогрев отсутствует $\left(I_{0}\right)$, а также порогового тока с учетом саморазогрева $\left(I_{\text {th }}\right)$. В области относительно больших диаметров $(D>40$ мкм для рассматриваемого случая) влияние са- 


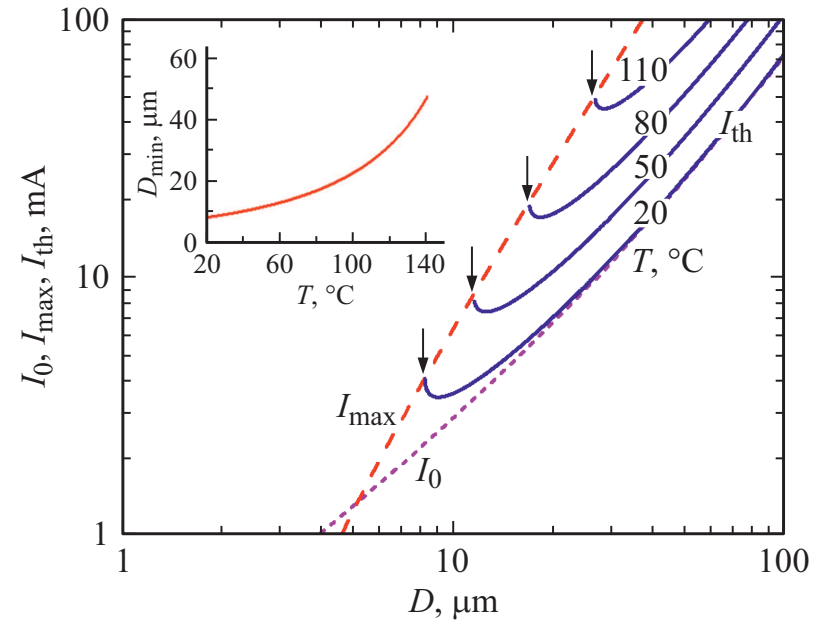

Рис. 4. Зависимость от диаметра порогового тока с учетом саморазогрева (сплошные линии) при различных температурах окружающей среды, а также максимального тока генерации (штриховая линия) и порогового тока в отсутствие саморазогрева при $20^{\circ} \mathrm{C}$ (точечная линия). Стрелки: минимальный диаметр микролазера при данной температуре. На вставке зависимость минимального размера микродиска от температуры.

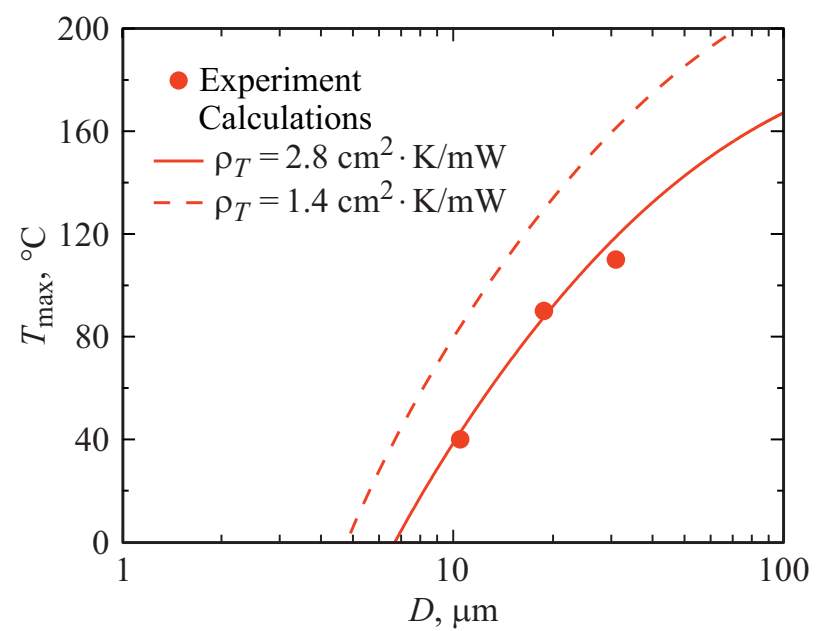

Рис. 5. Зависимость от диаметра предельной температуры генерации: символы - эксперимент, линии — расчет.

моразогрева невелико, так что $I_{\text {th }} \approx I_{0}$. При уменьшении диаметра вклад саморазогрева в пороговый ток увеличивается, что первоначально ведет к замедлению снижения $I_{\text {th }}$. При дальнейшем уменьшении $D$ пороговый ток достигает своего минимального значения $\sim 3 \mathrm{MA}$, после чего начинает возрастать. Когда значение $I_{\text {th }}$ сравнивается с $I_{\max }$, лазерная генерация прекращается. Это происходит при некотором наименьшем диаметре $\left(D_{\min }\right)$ микродиска. В рассматриваемом случае он составил $\sim 8.2$ мкм. Иными словами, при температуре окружающей среды $20^{\circ} \mathrm{C}$ достижение режима лазерной генерации в непрерывном режиме в микродисках, размер которых меньше этого диаметра, невозможно.
При увеличении $T$ пороговый ток возрастает. Вследствие этого точка пересечения кривой $I_{\text {th }}(D)$ с не зависящей от температуры кривой $I_{\max }(D)$ сдвигается вправо (рис. 4). Таким образом, минимальный диаметр микродиска, при котором возможна лазерная генерация, увеличивается с ростом температуры окружающей среды. Эта зависимость показана на вставке к рис. 4. Например, для того чтобы лазерная генерация была возможна при $T=110^{\circ} \mathrm{C}$, диаметр должен быть не менее 26.6 мкм.

На рис. 5 символами показана максимальная температура, при которой в микролазерах рассматриваемого типа наблюдалась лазерная генерация в непрерывном режиме (данные работы [10]). Сплошной линией показана расчетная зависимость предельной рабочей температуры микролазера $T_{\max }$ от его диаметра. Как видно, наблюдается хорошее совпадение результатов в области диаметров 10-30 мкм, для которой имеются экспериментальные данные. Для меньших диаметров расчет предсказывает резкое уменьшение предельной рабочей температуры вплоть до отрицательных температур (по шкале Цельсия) для $D<6.5$ мкм.

\section{5. Обсуждение}

Входящее в (7a) отношение электрического и теплового сопротивлений $R_{S} / R_{T}$ микролазера меняется

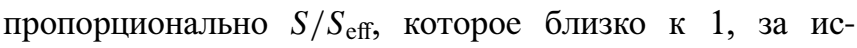
ключением самых малых диаметров, таких что $D \approx \delta$. Таким образом, зависимость тока $I_{\max }$ от диаметра определяется стоящим в знаменателе (7а) значением $R_{S}$, т. е. $I_{\max } \propto(D-\delta)^{2} \approx D^{2}$ (штриховая линия на рис. 4). Следовательно, значение $\Delta T_{\max }$ остается приблизительно постоянным. В то же время $I_{0}$ меняется с диаметром микролазера более сложным образом, что отражает изменение относительного вклада различных механизмов рекомбинации - в области больших диаметров $(D>40$ мкм) преобладает рекомбинация в объеме, а в области малых диаметров $(D<30$ мкм) доминирует безызлучательная рекомбинация на боковых стенках, т.е. $I_{0} \propto D$. Более быстрое $\left(\propto D^{2}\right)$ уменьшение $I_{\max }$ и является, в соответствии с (7б), причиной снижения предельной температуры генерации при уменьшении размера микродиска.

Эффективным способом повышения рабочей температуры микролазера, кроме подавления безызлучательной рекомбинации на боковых стенках микрорезонатора, является улучшение отвода тепла от активной области. Как свидетельствуют результаты расчетов, снижение удельного теплового сопротивления в 2 раза (до $1.4 \cdot 10^{-3} \mathrm{~cm}^{2} \cdot \mathrm{K} / \mathrm{BT}$ ) позволило бы увеличить рабочую температуру на $\sim 40^{\circ} \mathrm{C}$, а минимальный диаметр, при котором лазерная генерация была бы возможна при комнатной температуре, уменышился бы до 5.5 мкм (штриховая линия на рис. 5). 


\section{6. Заключение}

Таким образом, исследовано влияние саморазогрева микродискового лазера на его пороговый ток. Показано, что увеличение тока инжекции рано или поздно вызывает такой рост температуры активной области, что лазерная генерация становится невозможной. Получены аналитические выражения, которые позволяют вычислить максимальный ток, вплоть до которого сохраняется лазерная генерация, и предельную температуру лазерной генерации в непрерывном режиме. Проанализировано влияние размера микролазера на пороговотемпературные характеристики и показано, что предельная температура генерации резко снижается при уменьшении диаметра, делая невозможной генерацию при комнатной температуре. Для использованного в расчетах набора параметров прибора это происходит при диаметре $\sim 8$ мкм. Несмотря на то что полученные расчетные значения соответствуют параметрам, извлеченным для микродисковых лазеров конкретного типа (соответствующего экспериментальным данным, полученным для микродисковых лазеров с активной областью в виде квантовых ям-точек InGaAs), общие выводы настоящей работы и сама модель применимы для любых типов микролазеров.

\section{Финансирование работы}

Работа выполнена при поддержке проекта Российского научного фонда 19-72-30010.

\section{Конфликт интересов}

Авторы заявляют, что у них нет конфликта интересов.

\section{Список литературы}

[1] A.F.J. Levi, R.E. Slusher, S.L. McCall, T. Tanbun-Ek, D.L. Coblentz, S.J. Pearton. Electron. Lett., 28, 1010 (1992).

[2] T. Baba, M. Fujita, A. Sakai, M. Kihara, R. Watanabe. IEEE Photon. Technol. Lett., 9, 878 (1997).

[3] B. Gayral, J.M. Gerard, A. Lemaitre, C. Dupuis, L. Manin, J.L. Pelouard. Appl. Phys. Lett., 75, 1908 (1999).

[4] H. Cao, J.Y. Xu, W.H. Xiang, Y. Ma, S.-H. Chang, S.T. Ho, G.S. Solomon. Appl. Phys. Lett., 76, 3519 (2000).

[5] Y. Wan, J. Norman, Q. Li, M.J. Kennedy, D. Liang, C. Zhang, D. Huang, Z. Zhang, A.Y. Liu, A. Torres, D. Jung, A.C. Gossard, E.L. Hu, K.M. Lau, J.E. Bowers. Optica, 4, 940 (2017).

[6] N. Kryzhanovskaya, E. Moiseev, Yu. Polubavkina, M. Maximov, M. Kulagina, S. Troshkov, Yu. Zadiranov, Yu. Guseva, A. Lipovskii, M. Tang, M. Liao, J. Wu, S. Chen, H. Liu, A. Zhukov. Optics Lett., 42, 3319 (2017).

[7] Y. Wan, D. Inoue, D. Jung, J.C. Norman, C. Shang, A.C. Gossard, J.E. Bowers. Photon. Res., 6, 776 (2018).

[8] S. Zhu, B. Shi, Q. Li, K.M. Lau. Opt. Express, 26, 14514 (2018).
[9] N.V. Kryzhanovskaya, E.I. Moiseev, Yu.V. Kudashova, F.I. Zubov, A.A. Lipovskii, M.M. Kulagina, S.I. Troshkov, Yu.M. Zadiranov, D.A. Livshits, M.V. Maximov, A.E. Zhukov. Electron. Lett., 51, 1354 (2015).

[10] E. Moiseev, N. Kryzhanovskaya, M. Maximov, F. Zubov, A. Nadtochiy, M. Kulagina, Yu. Zadiranov, N. Kalyuzhnyy, S. Mintairov, A. Zhukov. Optics Lett., 43, 4554 (2018).

[11] Y. Wan, J. Norman, Q. Li, M.J. Kennedy, D. Liang, C. Zhang, D. Huang, A.Y. Liu, A. Torres, D. Jung, A.C. Gossard, E.L. Hu, K.M. Lau, J.E. Bowers. „CLEO: Applications and Technology, (San Jose, CA, USA, 2017) p. JTh5C.3.

[12] J.W. Scott, R.S. Geels, S.W. Corzine, L.A. Coldren. J. Lightwave Technol., 29, 1295 (1993).

[13] A. Al-Omari, K. Lear. IEEE Photon. Technol. Lett., 17, 1767 (2005).

[14] P.P. Baveja, B. Kogel, P. Westbergh, J.S. Gustavsson, A. Haglund, D.N. Maywar, G.P. Agrawal, A. Larsson. Opt Express, 19, 15490 (2011).

[15] S.A. Mintairov, N.A. Kalyuzhnyy, M.V. Maximov, A.M. Nadtochiy, S. Rouvimov, A.E. Zhukov. Electron. Lett., 51, 1602 (2015).

[16] N.V. Kryzhanovskaya, E.I. Moiseev, F.I. Zubov, A.M. Mozharov, M.V. Maximov, N.A. Kalyuzhnyy, S.A. Mintairov, M.M. Kulagina, S.A. Blokhin, K.E. Kudryavtsev, A.N. Yablonskiy, S.V. Morozov, Yu. Berdnikov, S. Rouvimov, A.E. Zhukov. Photon. Res., 7, 664 (2019).

[17] N.V. Kryzhanovskaya, E.I. Moiseev, F.I. Zubov, A.M. Mozharov, M.V. Maximov, N.A. Kalyuzhnyy, S.A. Mintairov, Yu.A. Guseva, M.M. Kulagina, S.A. Blokhin, Yu. Berdnikov, A.E. Zhukov. J. Appl. Phys., 126, 063107 (2019).

[18] Ф.И. Зубов, Э.И. Моисеев, Г.О. Корнышов, Н.В. Крыжановская, Ю.М. Шерняков, А.С. Паюсов, М.М. Кулагина, Н.А. Калюжный, С.А. Минтаиров, М.В. Максимов, А.Е. Жуков. Письма ЖТФ, 45 (19), 37 (2019).

Редактор А.Н. Смирнов

\section{Lasing temperature limit of a microdisk laser}

A.E. Zhukov' ${ }^{1}$, N.V. Kryzhanovskaya1 1', E.I. Moiseev' ${ }^{2}$, M.M. Kulagina ${ }^{3}$, S.A. Mintairov ${ }^{3}$, N.A. Kalyuzhnyy ${ }^{3}$, A.M. Nadtochiy ${ }^{2}$, M.V. Maximov ${ }^{2}$

${ }^{1}$ National Research University Higher School of Economics, 190008 St. Petersburg, Russia

${ }^{2}$ Alferov St. Petersburg Academic University, 194021 St. Petersburg, Russia

${ }^{3}$ loffe institute,

194021 St. Petersburg, Russia

Abstract A model is developed that allows one to analytically determine the threshold current of a microdisk laser taking into account its self-heating as a function of the ambient temperature and the microlaser diameter. It is shown that there exists a minimal diameter of a microdisk caused by self-heating, up to which it is possible to achieve continuous-wave lasing at a given temperature. Another manifestation of self-heating effect is the existence of a maximum operating temperature, which is the lower the smaller the diameter of the microlaser. Reasonable agreement between the predictions of the model and the available experimental data is shown. 